\title{
Prevalência de fluorose dentária em escolares de Marinópolis, São Paulo
}

\author{
Prevalence of dental fluorosis in school children \\ from Marinópolis, São Paulo
}

I onei de Maria Gomes Brandão 1

Arsenio Sales Peres 1

Nemre Adas Saliba 1

Suzely Adas Sali ba Moimaz 1

1 Faculdade de Odontol ogia
de Araçatuba, Universidade
Estadual Paulista.
C. P. 341, Araçatuba, SP
16015-050, Brasil.
iocesar@uol.com.br
arsenio@usp.br
nemre@foa.unesp.br
sasaliba@oa.unesp.br

\begin{abstract}
The purpose of this study was to determine the frequency and severity of dental fluorosis in 5 to 12 and 15 year-old school children in the city of Marinópolis, São Paulo, Brazil, with the purpose of obtaining a basel ine data for the monitoring dental fluorosis in this population. All of the school children in the both sexes, in the mentioned ages, enrolled in educational institutions and having the pre requirement condition of to live in Marinópolis from their birth were involved, totaling 320 school children. The exams were made by one examiner previously calibrated to use the Dean Index. According to the results, the frequency of dental flu orosis in the studied group was $17.2 \%$, however just consi dering the fluorosis levels that determine aesthetic compromising (mild, moderate and severe), this percentile was $7.19 \%$. The predominant level was the very mild (10.0\%) followed for the mild (5.3\%), moderate (1.3\%) and severe (0.6\%). Concluding that the dental fluorosis in the studied population doesn't constitute in a problem with wide dimensions, however, subsequent studies are necessary in the attempt of identifying the causes of moderate and severe fluorosis cases.
\end{abstract}

Key words Oral Health; School Health; Fluoride Poisoning; Child Health; Epi demiology

Resumo O objetivo deste estudo foi determinar a freqüência e severi dade da fluorose dentária em escolares de 5 a 12 e de 15 anos de idade da ci dade de Marinópolis, São Paulo, Brasi l, com o propósito de se obter um banco de dados-base inicial para o monitoramento da fluorose dentária nesta população. Foram envol vi dos todos os escolares de ambos os sexos, nas i dades citadas, matriculados nas insti tui ções de ensino da referida ci dade e tendo como pré requisito a condição de resi direm em Marinópolis desde o nascimento, total izando 320 escolares. Os exames foram realizados por um examinador previamente calibrado para a aplicação do Índice de Dean. De acordo com os resultados, a prevalência de fluorose dentária no grupo estudado foi de 17,2\%; no entanto, consi derando apenas os graus de fluorose que determi nam comprometi mento estético (leve, moderado e severo), o percentual foi de 7,19\%. O grau predominante foi o muito leve $(10,0 \%)$ segui do pel os graus leve (5,3\%), moderado (1,3\%) e severo $(0,6 \%)$. Conclui-se quea fluorose dentária na população estudada não se constitui em problema de amplas dimensões; estudos posteriores, contudo, são necessários, a fim de identificar as causas da presença de casos de fluorose moderada e severa.

Palavras-chave Saúde Bucal; Saúde Escolar; Intoxicação por Flúor; Saúde Infantil; Epidemiologia 


\section{Introdução}

A fluorose dentária é considerada um distúrbio específico de formação dentária, causada por excessiva ingestão de flúor durante o respectivo período (Möller, 1982; Murray, 1986). A severidade e a distribuição da fluorose dependem da concentração e duração da exposição do flúor, do estágio de atividade dos ameloblastos e da suscetibilidade individual (Horowitz, 1986; Limeback, 1994).

A prevalência de fluorose dentária aumentou consideravel mente em muitas partes do mundo nos anos 90 (Ahokas et al., 1999). Como efeito indesejável desse panorama, pode-se citar o aumento do risco de defeitos de esmalte, de comprometimento estético perceptível ao público leigo, apresentando implicações psicológicas, financei ras e comportamentais a estes, além da possibilidade de colocar em risco a aceitação pública do uso de fluoretos (Ahokas et al., 1999; Clark et al., 1993).

Considerando que o conhecimento da realidade epidemiológica é fundamental para o planejamento, estruturação, execução e avaliação de toda e qualquer ação relacionada com serviços de saúde, no tocante às condições de saúde bucal de dada população é imperiosa a realização de estudos visando ao monitoramento da distribuição dos principais problemas de saúde bucal na população e de suas causas (Souza \& Bergamashi J r., 1999).

A cidade de Marinópolis, São Paulo, segundo projeções realizadas pela Fundação SEADE (Fundação Sistema Estadual de Análise de Dados), com base no Censo Demográfico-1991, contava com uma população de 2.192 habitantes em 2000. A população urbana é de 1.644 habitantes $(75,00 \%)$ e a rural de 548 habitantes $(25,00 \%)$. Quase que a totalidade da população $(98,84 \%)$ tinha acesso ao abastecimento de água em 1991 (Fundação SEADE, 2000).

A população dispõe do sistema de fluoretação das águas de abastecimento público $(0,7$ ppm de flúor), iniciado em 1983, sendo efetuado e controlado desde então exclusivamente pela Companhia de Saneamento Básico do Estado de São Paulo (SABESP). De acordo com informação da autoridade local na área de saúde bucal, os escolares de 7 a 14 anos de idade realizam, de forma regular, bochechos semanais com solução fluoretada desde 1981.

No âmbito da saúde bucal, não existiam dados epidemiológicos oficiais da referida população até o momento, o que justifica este estudo, que teve como objetivo obter dados que permitissem determinar a prevalência e severidade da fluorose dentária nos escolares de 5 a
12 e de 15 anos de idade da cidade de Marinópolis, com o propósito de se obter um banco de dados-base inicial para o monitoramento da fluorose dentária nesta população.

\section{Material e métodos}

- População de estudo: foi composta por todos os escolares matriculados nas instituições de ensino do Município de Marinópolis.

- População de referência: seguindo orientação da Organização Mundial da Saúde (OMS), optou-se por envolver no estudo escolares de ambos os sexos, de 5 a 12 e de 15 anos de idade, matriculados nas instituições de ensino da referida cidade e tendo como pré-requisito a condição de residirem em Marinópolis desde o nascimento.

- Instrumento de coleta de dados: o índice utilizado foi o de Dean, que classifica a fluorose dentária nos seguintes graus: $0=$ normal; $1=$ questionável; 2 =muito leve; 3 =leve; 4 =moderado e 5 =severo, sendo aplicado com base nos critérios preconizados pela OMS (1999). Os exames foram realizados por um único examinador, devidamente treinado e calibrado (OMS, 1999). No processo de calibração, foi feita a avaliação da concordância dos resultados - por meio da aplicação da estatística Kappa (k) (Kramer \& Feinstein, 1981), tanto entre o examinador e um padrão ouro $(\mathrm{k}=0,81)$, assim como o examinador e o intra-examinador $(k=0,83)$, sendo esta última repetida durante o trabalho de campo $(k=0,81)$. Tais valores são correspondentes a uma concordância quase perfeita entre os examinadores.

Os exames foram realizados sob luz natural, sendo utilizadas cadeiras e carteiras escolares, com auxílio de espel hos bucais planos (previamente esterilizados).

- Processamento de dados e análise estatística: o programa Epi-Info, versão 5.0 (CDC, 1990/ 1991), de domínio público, desenvolvido para a OMS, foi utilizado para montagem do banco de dados e a entrada das informações. Uma versão específica do programa EPIBUCO, criado no Epi-Info pelo Prof. Eymar Sampaio Lopes, da Faculdade de Odontologia de Bauru, Universidade de São Paulo (USP), foi empregado para processamento dos dados e análise estatística.

\section{Resultados e discussão}

Considerando o tamanho reduzido da população, optou-se pelo exame de todos os alunos 
nas idades de 5 a 12 e de 15 anos que residiam desde o nascimento na cidade de Marinópolis $(\mathrm{N}=320)$. Dessa forma, foram excluídos para os cálculos seguintes sete escolares que não preenchiam tal requisito. Em adição, somente foram examinados os escolares que tiveram prévio consentimento esclarecido pelos respectivos responsáveis. As informações referentes à população de estudo final estão apresentadas na Tabela 1.

Os dados relativos à fluorose dentária na população estudada são apresentados nas Tabelas 2 e 3. De acordo com os dados da Tabela 2, que representam a prevalência de fluorose dentária nos escolares examinados, observa-se que $17,2 \%$ dos mesmos apresentam tal condição.

A Tabela 3 apresenta a distribuição percentual dos escolares por idade e de acordo com os diversos graus de fluorose. Observa-se que $17,2 \%$ apresentaram graus de fluorose variando de 2 a 5, sendo $10,0 \%$ de grau 2; 5,3\% de grau 3; $1,3 \%$ de grau 4 e $0,6 \%$ de grau 5 .

Em 1998, a Secretaria de Estado da Saúde de São Paulo (1999), em parceria com a Faculdade de Saúde Pública, USP, real izou o Levantamento Epidemiológico em Saúde Bucal do Estado, no qual os 133 municípios foram classificados de acordo com as regiões de saúde (DIR) estabeleci das pela Secretaria de Estado da Saúde, o porte (pequeno, médio e grande) e quanto à disponibilidade de água fluoretada no núcleo urbano.

Considerando o grupo de idade de 12 anos, quando se comparam os resultados deste citado estudo para os municípios do Estado que possuem água fluoretada com os obtidos na cidade de Marinópolis, destaca-se o fato da fluorose afetar maior percentual de escolares em Marinópolis, nos graus 2, 3, 4 e 5 (Tabela 4).

Pereira (1996), investigando a prevalência de fluorose dentária em escolares de 12 a 14 anos de idade de Cesário Lange (1,4ppm de flúor na água), Piracicaba (0,7ppm de flúor na água) e Iracemápolis (<0,3ppm de flúor na água), obteve respectivamente os seguintes valores: $32,4 \%$; $16,9 \%$ e $4,2 \%$.

Campos et al. (1998), avaliando a prevalência de fluorose dentária em escolares de 8 a 12 anos de Brasília, Distrito Federal $(0,8 p p m$ de flúor na água), observaram que $14,64 \%$ da população estudada apresentavam tal enfermidade. No entanto, a prevalência de fluorose no grupo de 12 anos foi de 3,6\%, valor muito inferior ao observado no presente estudo (39,4\%), além do fato de não terem sido diagnosticados casos de fluorose severa.

Recentes estudos têm relatado que a prevalência e a severidade da fluorose dentária têm
Tabela 1

Número e porcentagem de escolares examinados, segundo idade e sexo. Marinópolis, São Paulo, 2000.

\begin{tabular}{|c|c|c|c|c|c|c|}
\hline \multirow[t]{3}{*}{ Idade } & \multicolumn{4}{|c|}{ Sexo } & \multicolumn{2}{|c|}{ Total } \\
\hline & \multicolumn{2}{|c|}{ Feminino } & \multicolumn{2}{|c|}{ Masculino } & & \\
\hline & $n$ & $\%$ & $\mathrm{n}$ & $\%$ & $n$ & $\%$ \\
\hline 5 & 18 & 62,1 & 11 & 37,9 & 29 & 100,0 \\
\hline 6 & 28 & 60,9 & 18 & 39,1 & 46 & 100,0 \\
\hline 7 & 11 & 31,4 & 24 & 68,6 & 35 & 100,0 \\
\hline 8 & 21 & 50,0 & 21 & 50,0 & 42 & 100,0 \\
\hline 9 & 23 & 51,1 & 22 & 48,9 & 45 & 100,0 \\
\hline 10 & 17 & 58,6 & 12 & 41,4 & 29 & 100,0 \\
\hline 11 & 20 & 44,4 & 25 & 55,6 & 45 & 100,0 \\
\hline 12 & 12 & 36,4 & 21 & 63,6 & 33 & 100,0 \\
\hline 15 & 6 & 37,5 & 10 & 62,5 & 16 & 100,0 \\
\hline Total & 156 & 48,8 & 164 & 51,2 & 320 & 100,0 \\
\hline
\end{tabular}

Tabela 2

Prevalência de fluorose dentária em escolares. Marinópolis, São Paulo, 2000.

\begin{tabular}{crrrrr}
\hline \multirow{2}{*}{ Idade } & \multicolumn{4}{c}{ Prevalência de fluorose } & Total \\
& \multicolumn{2}{c}{ Ausência } & \multicolumn{2}{c}{ Presença } & \\
& $n$ & $\%$ & $n$ & $\%$ & \\
\hline 5 & 28 & 96,6 & 1 & 3,4 & 29 \\
6 & 43 & 93,5 & 3 & 6,5 & 46 \\
7 & 32 & 91,4 & 3 & 8,6 & 35 \\
8 & 39 & 92,8 & 3 & 7,2 & 42 \\
9 & 38 & 84,5 & 7 & 15,5 & 45 \\
10 & 15 & 51,7 & 14 & 48,2 & 29 \\
11 & 38 & 84,5 & 7 & 15,5 & 45 \\
12 & 20 & 60,6 & 13 & 39,4 & 33 \\
15 & 12 & 75,0 & 4 & 25,0 & 16 \\
Total & 265 & 82,8 & 55 & 17,2 & 320 \\
\hline
\end{tabular}

aumentado tanto em regiões abastecidas por água fluoretada, como nas que não possuem esse benefício (Clark, 1994; Lewis \& Banting, 1994). Esse aumento tem sido atribuído ao consumo de flúor proveniente de diversas outras fontes, tais como dentifrícios fluoretados, suplementos dietéticos contendo flúor e certos alimentos (Clark, 1994; Lewis \& Banting, 1994; Mann et al., 1990).

De acordo com Ahokas et al. (1999), os possíveis fatores de risco associados à fluorose dentária incluem: residir em região abastecida por água otimamente fluoretada, uso de suplementos de flúor, nível de flúor no dentifrício, 
Tabela 3

Número e percentual dos escolares, segundo a idade e os graus de fluorose. Marinópolis, São Paulo, 2000.

\begin{tabular}{|c|c|c|c|c|c|c|c|c|c|c|c|c|c|c|c|}
\hline \multirow[t]{3}{*}{ Idade } & \multicolumn{4}{|c|}{ Sem fluorose } & \multicolumn{8}{|c|}{ Com fluorose } & \multirow{2}{*}{\multicolumn{2}{|c|}{$\begin{array}{l}\text { Sem informação, } \\
\text { excluído }\end{array}$}} & \multirow[t]{3}{*}{ Total } \\
\hline & \multicolumn{2}{|c|}{$\begin{array}{c}0 \\
\text { Normal }\end{array}$} & \multicolumn{2}{|c|}{$\begin{array}{c}1 \\
\text { Questionável }\end{array}$} & \multicolumn{2}{|c|}{$\begin{array}{c}2 \\
\text { Muito Leve }\end{array}$} & \multicolumn{2}{|c|}{$\begin{array}{c}3 \\
\text { Leve }\end{array}$} & \multicolumn{2}{|c|}{$\begin{array}{c}4 \\
\text { Moderada }\end{array}$} & \multicolumn{2}{|c|}{$\begin{array}{c}5 \\
\text { Severa }\end{array}$} & & & \\
\hline & $\mathrm{n}$ & $\%$ & $\mathrm{n}$ & $\%$ & $\mathrm{n}$ & $\%$ & $\mathrm{n}$ & $\%$ & $\mathrm{n}$ & $\%$ & $\mathrm{n}$ & $\%$ & $\mathrm{n}$ & $\%$ & \\
\hline 5 & 28 & 96,6 & 0 & - & 1 & 3,4 & 0 & - & 0 & - & 0 & - & 0 & - & 29 \\
\hline 6 & 42 & 91,3 & 1 & 2,2 & 3 & 6,5 & 0 & - & 0 & - & 0 & - & 0 & - & 46 \\
\hline 7 & 32 & 91,4 & 0 & - & 2 & 5,7 & 1 & 2,9 & 0 & - & 0 & - & 0 & - & 35 \\
\hline 8 & 39 & 92,8 & 0 & - & 1 & 2,4 & 2 & 4,8 & 0 & - & 0 & - & 0 & - & 42 \\
\hline 9 & 37 & 82,3 & 1 & 2,2 & 5 & 11,1 & 2 & 4,4 & 0 & - & 0 & - & 0 & - & 45 \\
\hline 10 & 15 & 51,7 & 0 & - & 7 & 24,1 & 6 & 20,7 & 0 & - & 1 & 3,4 & 0 & - & 29 \\
\hline 11 & 38 & 84,5 & 0 & - & 5 & 11,1 & 2 & 4,4 & 0 & - & 0 & - & 0 & - & 45 \\
\hline 12 & 19 & 57,6 & 1 & 3,0 & 5 & 15,2 & 4 & 12,1 & 3 & 9,1 & 1 & 3,0 & 0 & - & 33 \\
\hline 15 & 12 & 75,0 & 0 & - & 3 & 18,6 & 0 & - & 1 & 6,4 & 0 & - & 0 & - & 16 \\
\hline Total & 262 & 81,9 & 3 & 0,9 & 32 & 10,0 & 17 & 5,3 & 4 & 1,3 & 2 & 0,6 & 0 & - & 320 \\
\hline
\end{tabular}

Tabela 4

Percentual de escolares de 12 anos, segundo o local e os graus de fluorose. Marinópolis, São Paulo, 2000.

\begin{tabular}{|c|c|c|c|c|c|c|c|c|}
\hline \multirow[t]{2}{*}{ Idade } & \multicolumn{2}{|c|}{ Sem fluorose } & \multicolumn{4}{|c|}{ Com fluorose } & \multirow{2}{*}{$\begin{array}{l}\text { Sem informação, } \\
\text { excluído }\end{array}$} & \multirow[t]{2}{*}{$\mathrm{n}$} \\
\hline & $\begin{array}{c}0 \\
\text { Normal }\end{array}$ & $\begin{array}{c}1 \\
\text { Questionável }\end{array}$ & $\begin{array}{c}2 \\
\text { Muito Leve }\end{array}$ & $\begin{array}{c}3 \\
\text { Leve }\end{array}$ & $\begin{array}{c}4 \\
\text { Moderada }\end{array}$ & $\begin{array}{c}5 \\
\text { Severa }\end{array}$ & & \\
\hline Marinópolis & 57,6 & 3,0 & 15,2 & 12,1 & 9,1 & 3,0 & - & 33 \\
\hline Estado* & 75,1 & 9,7 & 11,7 & 2,3 & 0,8 & 0,1 & 0,3 & 5.668 \\
\hline
\end{tabular}

* Valores correspondentes aos municípios selecionados do Estado que possuem flúor na água de abastecimento público.

Fonte: Secretaria de Estado da Saúde de São Paulo (1999).

idade precoce de início da escovação com dentifrício, alta freqüência de escovação com dentifrício/ingestão de dentifrício, desmame precoce, uso prolongado de fórmulas infantis.

Segundo Dean (1938), 10\% das pessoas nascidas e criadas em uma comunidade com água otimamente fluoretada demonstrariam sinais de formas brandas de fluorose, sendo este, de acordo com o autor, o percentual sem significado em termos de saúde pública.

Com relação a este estudo, merece destaque o fato de terem sido encontrados, na cidade de Marinópolis, portadores de fluorose moderada e severa (Tabela 3).

Segundo McDonagh et al. (2000), ainda atualmente, quando se estabelece o percentual de prevalência de fluorose em uma dada popuIação, considera-se que qual quer criança que apresente algum grau de fluorose é classificada como uma portadora dessa alteração. Parecenos mais lógica a proposta citada no estudo de
Hawley et al. (1996), segundo a qual apenas as crianças que apresentassem graus de fluorose classificados como de "comprometimento estéti co" seriam consideradas nos cál culos para a determinação da prevalência de fluorose dentária. Considerando o índice de Dean, os graus leve, moderado e severo assim se enquadrariam (McDonagh et al., 2000).

Dessa forma, associando-se tais consi derações com os resultados obtidos neste estudo pode-se constatar que, da população examinada $(\mathrm{N}=320), 7,19 \%(\mathrm{n}=23)$ das crianças apresentaram graus de fluorose variando do leve ao severo, sendo este o percentual de portadores de fluorose com "comprometimento estético" (Hawley et al., 1996), nesta referida população.

De acordo com Campos et al. (1998), uma vez observada a fluorose dentária em determinada população, estudos posteriores precisam ser realizados na tentativa de identificar suas causas. Ou seja, os estudos descritivos como 
este são importantes e imprescindíveis, no entanto, com bases apenas nestes resultados, nada se pode afirmar a respeito de quais fatores estariam levando à situação descrita.

\section{Conclusões}

De acordo com os resultados obtidos, pode-se concluir que: (1) a prevalência de fluorose dentária nos escolares estudados foi de 17,2\%; (2) considerando apenas os graus leve, moderado e severo, o percentual de portadores de fluorose com comprometimento estético foi de 7,19\%; (3) o grau predominante foi o muito leve $(10,0 \%)$, seguido pelo grau leve $(5,3 \%)$, moderado $(1,3 \%)$ e severo $(0,6 \%) ;(4)$ a fluorose dentária na população estudada não se constitui em problema de amplas dimensões, no entanto estudos posteriores são necessários na tentativa de identificar as causas da presença de casos de fluorose moderada e severa; (5) recomenda-se heterocontrole do teor de flúor nas águas de abastecimento público.

\section{Referências}

AHOKAS, J. T.; DEMOS, L.; DONOHUE, D. C.; KILLALEA, S.; MCNEIL, J. \& RIX, C. J., 1999. Review of Water Fluoridation and Fluoride Intake from Discretionary Fluoride Supplements. Melbourne: National Health and Medical Research Council.

CAM POS, D. L.; FARIAS, D. C.; TOLEDO, O. A. \& BEZERRA, A. C. B., 1998. Prevalência de fluorose dentária em escolares de Brasília. Revista de Odontologia da USP, 12:225-230.

CDC (Centers for Disease Control and Prevention), 1990/ 1991. Epi Info Version 5.01. Stone Mountain: CDC.

CLARK, D. C., 1994. Trends in the prevalence of dental fluorosis in North America. Community Dentistry and Oral Epidemiology, 22:148-152.

CLARK, D. C.; HANN, H. J.; WILLIAMSON, M. F. \& BERKOWITS, J., 1993. Aesthetic concerns of children and parents in relation to different classifications of the Tooth Surface Index of Fluorosis. Community Dentistry and Oral Epidemiology, 21:360-364.

DEAN, T. H., 1938. Endemic fluorosis and its relation to dental caries. Public Health Reports, 53:14431452.

FUNDAÇÃO SEADE (Fundação Sistema Estadual de Análise de Dados), 2000. Informações dos Municípios Paulistas. 15 Novembro 2000 বhttp:// www. seade.gov.br/lingcv98/?n1=15>.

HAWLEY, G. M.; ELLWOOD, R. P. \& DAVIES, R. M., 1996. Dental caries, fluorosis and the cosmetic implications of different TF scores in 14-year old adolescents. Community Dental Health, 13:189192.

HOROWITZ, H. S., 1986. Index of measuring dental fluorosis. Journal of Public Health Dentistry, 46:179-183.

KRAMER, M. S. \& FEINSTEIN, A. R., 1981. Clinical biostatistics: The biostatistics of concordance. Clinical Pharmacology \& Therapeutics, 29:454-459.

LEWIS, D. W., 1994. Water fluoridation: Current effectiveness and dental fluorosis. Community Dentistry and Oral Epidemiology, 22:153-158.
LIMEBACK, H., 1994. Enamel formation and the effects of fluoride. Community Dentistry and Oral Epidemiology, 22:144-147.

MANN, J.; MAHMOUD, W.; ERNEST, M.; SGAN-COHEN, H.; SHOSHAN, N. \& GEDALIA, I., 1990. Fluorosis and dental caries in 6-8-years-old children in a $5 \mathrm{ppm}$ fluoride area. Community Dentistry and Oral Epidemiology, 18:77-79.

MCDONAGH, M.; WHITING, P.; BRADLEY, M.; COOPER, J.; SUTTON, A.; CHESTNUTT, I.; MISSO, K.; WILSON, P.; TREASURE, E. \& KLEIJNEN, J., 2000. A Systematic Review of Public Water Fluoridation. York: National Health Services Centre for Reviews and Dissemination.

MÖLLER, I. J., 1982. Fluorides and dental fluorosis. International Dental Journal, 32:135-147.

MURRAY, J. J., 1986. Appropriate Use o Fluoride for Human Health. Genebra: World Health Organizaton.

OMS (Organização Mundial da Saúde), 1999. Levantamentos Básicos em Saúde Bucal. 4ạ Ed. São Paulo: Santos Livraria.

PEREI RA, A. C., 1996. Comparação entre Três Índices de Fluorose Dentária na Dentição Permanente, Observados em Escolares de 12 a 14 Anos de Idade, Residentes em Áreas com Diferentes Concentrações deFlúor nas Águas de Consumo. Tese de Doutorado, São Paulo: Faculdade de Saúde Pública, Universidade de São Paulo.

SECRETARIA DE ESTADO DA SAÚDE DE SÃO PAULO, 1999. Levantamento Epidemiológico em Saúde Bucal: Estado de São Paulo, 1998. São Paulo: Secretaria de Estado da Saúde de São Paulo/ Faculdade de Saúde Pública, Universidade de São Paulo.

SOUZA, R. A. A. R \& BERGAMASHI Jr., E., 1999. Estudo da Prevalência de Cárie Dentária eFluorose no Município de Araçatuba-SP. Araçatuba: Faculdade de Odontologia de Araçatuba, Universidade Estadual Paulista.

Recebido em 22 de março de 2001

Versão final reapresentada em 16 de outubro de 2001 Aprovado em 28 de dezembro de 2001 